\title{
Analyse chimique immédiate des matières grasses par capteur spectrophotométrique
}

Oléagineux, Corps Gras, Lipides. Volume 10, Numéro 4, 248-50, JUILLET-AOÛT 2003, Innovation dans l'analyse et le traitement des donné\#233;es

Auteur(s) : Marc MEURENS, Université catholique de Louvain Biochimie de la Nutrition -- Croix du Sud 2 (8) -- 1348 Louvain-la-Neuve-- Belgique Tel : +3210473726 Fax : +3210473728 $<$ meurensbnut.ucl.ac.be $>$.

Author(s) : Marc MEURENS

Résumé : Les techniques spectrophotométriques permettent de déterminer instantanément la concentration de différentes espèces chimiques sans préparation ni transformation des échantillons. L'application de ces techniques et notamment de la spectroscopie proche infrarouge en contrôle de qualité des industries alimentaires est développée par de nombreux laboratoires et entreprises depuis plus de vingt ans. Les performances analytiques de nouveaux spectrophotomètres en mesure tant d'émission fluorescente et Raman que d'absorption proche infrarouge sont présentées ici, afin de montrer comment ces instruments répondent aux besoins d'analyse rapide et de contrôle de qualité en ligne dans le domaine oléicole. Dotés de fibres optiques et de détecteurs à couplage de charge, les spectrophotomètres de la dernière génération appelés capteurs spectrophotométriques ou spectrocapteurs s'avèrent convenir à l'analyse chimique immédiate et non destructive des matières grasses avec la détermination de paramètres comme les teneurs en lipides totaux et en acides gras libres pour la spectrométrie proche infrarouge, les teneurs en différents polyphénols et en chlorophylle pour la spectrofluorimétrie et les teneurs en acides gras à doubles liaisons en configuration trans pour la spectrométrie Raman.

Summary : The spectrophotometric techniques allow to determine instantaneously the concentration of chemical species without sample preparation and transformation. The application of these techniques, notably the near infrared spectroscopy in quality control of food industry has been developed by numerous laboratories and companies for more than twenty years. The analytical performances of new spectrophotometers in measurement of fluorescent and Raman emission as well as near infrared absorption are presented here in order to explain how these instruments answer the needs of fast analysis and on-line quality control in the oils and fats domain. Equipped with fiber optics and coupled charge device detectors, the last generation of spectrophotometers named spectrophotometric sensors or spectrosensors suit the immediate and non-destructive chemical analysis of fats and oils with the determination of parameters such as total lipids, free fatty acids for the near infrared spectrometry, polyphenols and chlorophyl for the spectrofluorimetry and trans fatty acids for the Raman spectroscopy.

Mots-clés : spectroscopie proche infrarouge, Raman, spectrofluorimétrie, huiles, graisses, contrôle de qualité en ligne.

Keywords : near infrared, Raman spectroscopy, spectrofluorimetry, fats, oils, chemical analysis, online quality control. 


\section{ARTICLE}

\section{Auteur(s) : Marc MEURENS}

Université catholique de Louvain Biochimie de la Nutrition - Croix du Sud 2 (8) - 1348 Louvain-laNeuve - Belgique

Tel : +3210473726

Fax : +3210473728

<meurens@bnut.ucl.ac.be>

Les spectrophotomètres dispersifs ont évolué ces dernières années depuis les instruments à réseaux mobiles et détecteurs monocanaux vers les instruments à réseaux fixes et barrettes de diodes. Dans la zone spectrale proche infrarouge, les spectrophotomètres présentent effectivement cette évolution avec des détecteurs du type barrette de diodes en silicium ou dispositif à couplage de charge (CCD) pour les longueurs d'onde comprises entre 700 et 1100 nanomètres (nm), du type barrette de diodes en arséniure d'indium-gallium (InGaAs) pour les longueurs d'onde comprises entre 1100 et $2500 \mathrm{~nm}$. Les fibres optiques en verre valables pour le proche infrarouge, le visible et I'ultraviolet permettent d'assembler les spectrophotomètres par modules où la source lumineuse, la cellule de mesure et le détecteur peuvent être séparés de plusieurs mètres, ce qui permet de construire des appareils convenant très bien aux conditions du contrôle de qualité en ligne.

\section{Spectrométrie proche infrarouge}

Un petit spectrophotomètre OCEAN OPTICS USB2000 à fibre optique couvrant la plage spectrale visible et proche infrarouge de 400 à 1100 nanomètres $(\mathrm{nm})$ est présenté à la figure 1 . Cet instrument est doté d'un détecteur CCD beaucoup plus sensible que les détecteurs à barrette de diodes en silicium. Le calibrage d'un petit spectrophotomètre de ce type a été réalisé pour la détermination des lipides totaux et des acides gras libres sur une quarantaine d'échantillons d'huile de palme et de palmiste. Les spectres d'absorbance $(\log 1 / T)$ proche infrarouge calculée à partir de la transmission $(T=I t / l o)$ à travers une épaisseur de 5 centimètres d'eau, d'huile de palme et d'huile de palmiste sont montrés à la figure 2. Les résultats de calibrage obtenus avec la méthode de régression linéaire multiple MLR sont présentés dans le tableau 1. R est le coefficient de corrélation entre les résultats d'analyse par spectrométrie proche infrarouge et les données de référence. SEC est l'erreur standard de calibrage. CV est le coefficient de variation ou l'erreur standard relative rapportée à la concentration moyenne et exprimée en \%. La précision du calibrage est indiquée par une erreur relative inférieure ou égale à $5 \%$ pour les déterminations de l'eau et des acides gras libres de l'huile de palmiste et une erreur comprise entre de 6 et $11 \%$ pour les paramètres correspondants de l'huile de palme. Ces résultats permettent d'envisager l'utilisation du petit spectrophotomètres à fibre optique et détecteur CCD comme capteur dans le contrôle en ligne des matière grasses. 
Tableau 1. Résultats de calibrage du spectrophotomètre proche infrarouge $(700-1100 \mathrm{ml})$ à fibre optique et détecteur CCD pour le dosage de l'eau et des acides gras libres (AGL) dans les huiles de palme et de palmiste.

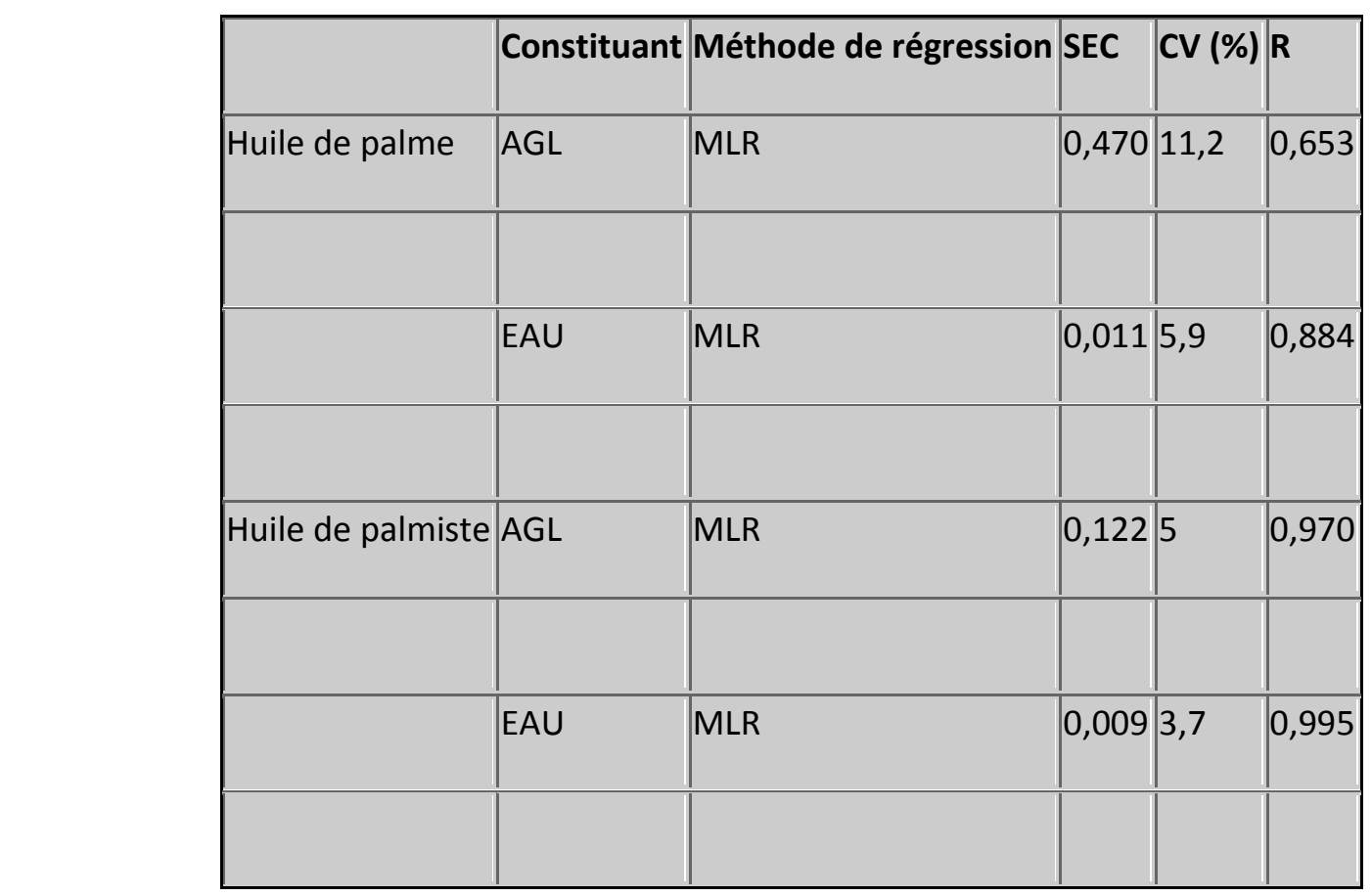

\section{Spectrofluorimétrie}

Le même spectrophotomètre a été testé en spectrofluorimétrie avec une source de lumièremonochromatique tel qu'un laser à argon SPECTRA - PHYSICS Stabilite 2017 réglé à $350 \mathrm{~nm}$ de longueur d'onde et $50 \mathrm{~mW}$ de puissance. Les spectres de la fluorescence induite par le laser sur de I'huile d'olive et de l'huile de noisette sont montrés à la figure 3. On peut observer que la fluorescence des deux huiles est tout à fait différente. Alors que l'huile de noisette émet de la fluorescence dans la zone spectrale entre 400 et $600 \mathrm{~nm}$, l'huile d'olive en émet principalement aux environs de $700 \mathrm{~nm}$. La fluorescence de l'huile de noisette est due surtout aux polyphénols, celle de I'huile d'olive est due essentiellement à la chlorophylle. La possibilité d'obtenir des spectres équivalents avec une source lumineuse moins coûteuse telle qu'une petite diode laser NICHIA NHVL3000 de $50 \mathrm{~mW}$ à la longueur d'onde de $400 \mathrm{~nm}$ permet de penser qu'un capteur constitué d'une simple diode laser violette et d'un petit spectrophotomètre à détecteur CCD pourrait convenir au contrôle en ligne du raffinage des huiles.

\section{Spectrométrie Raman}

Le même petit spectrophotomètre que celui utilisé pour la fluorimétrie pourrait servir aussi à mesurer la diffusion Raman qui, comme la fluorescence, est une réémission des photons de la lumière d'excitation à une longueur d'onde supérieure. Pour détecter l'effet Raman induit par un laser proche infrarouge avec un spectrophotomètre CCD (insensible aux longueurs d'onde supérieures à $1100 \mathrm{~nm}$ ), il faut utiliser une source d'excitation lumineuse monochromatique à une longueur d'onde inférieure à $800 \mathrm{~nm}$. Moyennant une excitation laser de $700 \mathrm{~mW}$ à $785 \mathrm{~nm}$, il est possible d'obtenir des spectres comme ceux des 80 échantillons de matière grasse laitière anhydre (MGLA) qui sont montrés à la figure 4. La zone spectrale comprise entre 1640 et $1680 \mathrm{~cm}^{-1}$ est présentée en fenêtre pour faire apparaître en détail les bandes caractéristiques des doubles liaisons 
cis $\left(1650 \mathrm{~cm}^{-1}\right)$ et trans $\left(1670 \mathrm{~cm}^{-1}\right)$ des acides gras insaturés. À partir de ces spectres, le calibrage d'un spectromètre Raman à transformée de Fourier a été réalisé (résultats non publiés) pour la détermination des acides gras trans de MGLA avec une erreur standard relative de moins de $10 \%$. Cette détermination paraît également faisable au moyen d'un petit spectrophotomètre CCD tel que celui qui a été présenté en spectrométrie proche infrarouge.

\section{Conclusion}

Au vu des quelques performances analytiques présentées ci-dessus, il apparaît que les petits spectrophotomètres visibles et proche infrarouges à détecteur $C C D$ peuvent répondre valablement aux exigences du contrôle de qualité en ligne dans les industries des matières grasses pour autant que les sources lumineuses et les composants opto-électroniques nécessaires à leur fonctionnement dans les conditions industrielles soient bien au point. Comme ces nouveaux instruments de contrôle sont automatisables et permettent une analyse chimique immédiate et non destructive, ils peuvent être qualifiés de capteurs spectrophotométriques ou spectrocapteurs. $\mathrm{n}$

\section{Illustrations}

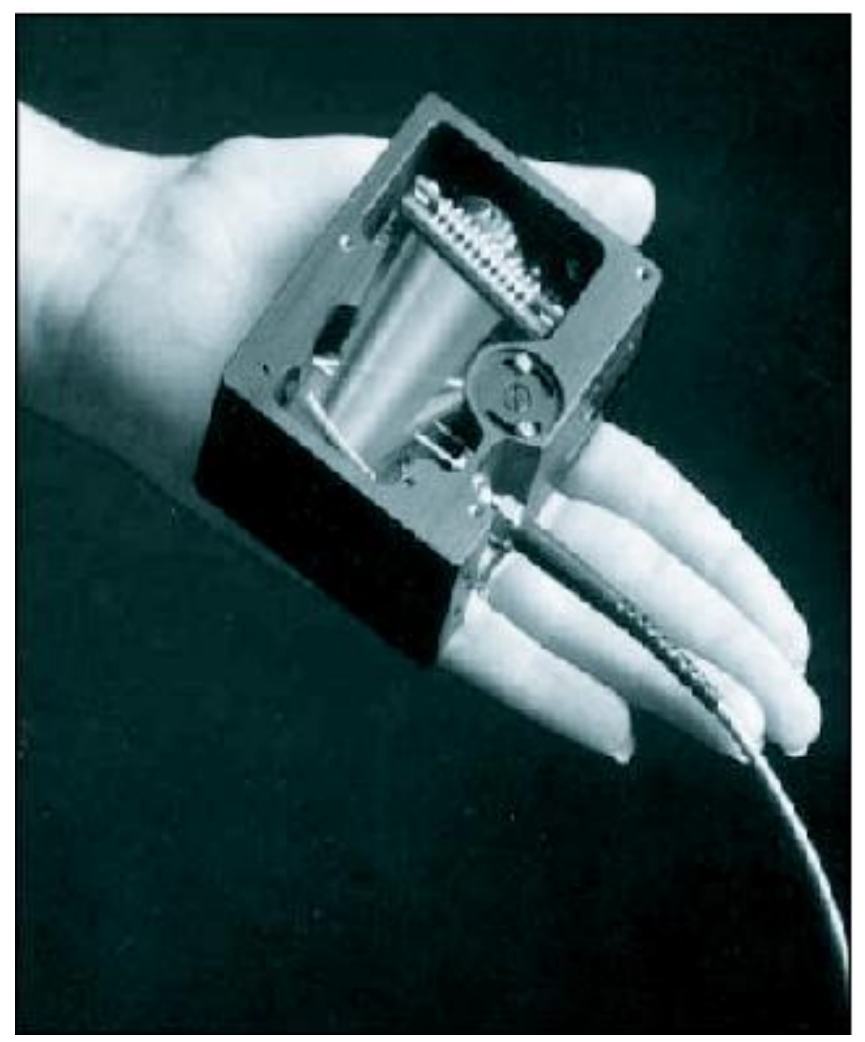

Figure 1. Photographie d'un petit spectrophotomètre OCEAN OPTICS USB2000 ouvert dans lequel la lumière visible et proche infrarouge amenée par une fibre optique est diffractée par un réseau fixe et dirigée vers un détecteur $C C D$. 


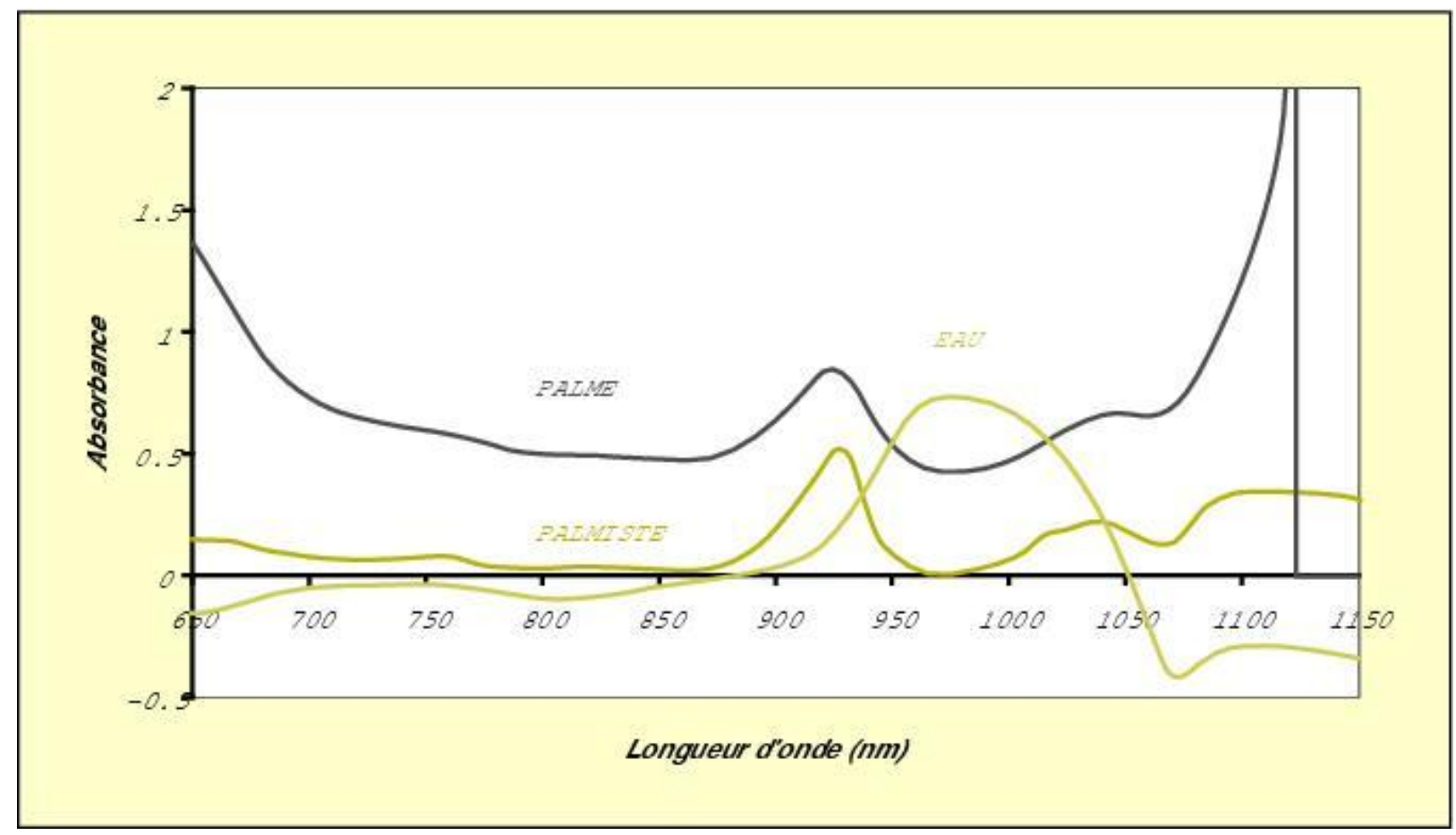

Figure 2. Spectres d'absorbance proche infrarouge à travers 5 centimètres d'eau, d'huile de palme et d'huile de palmiste.

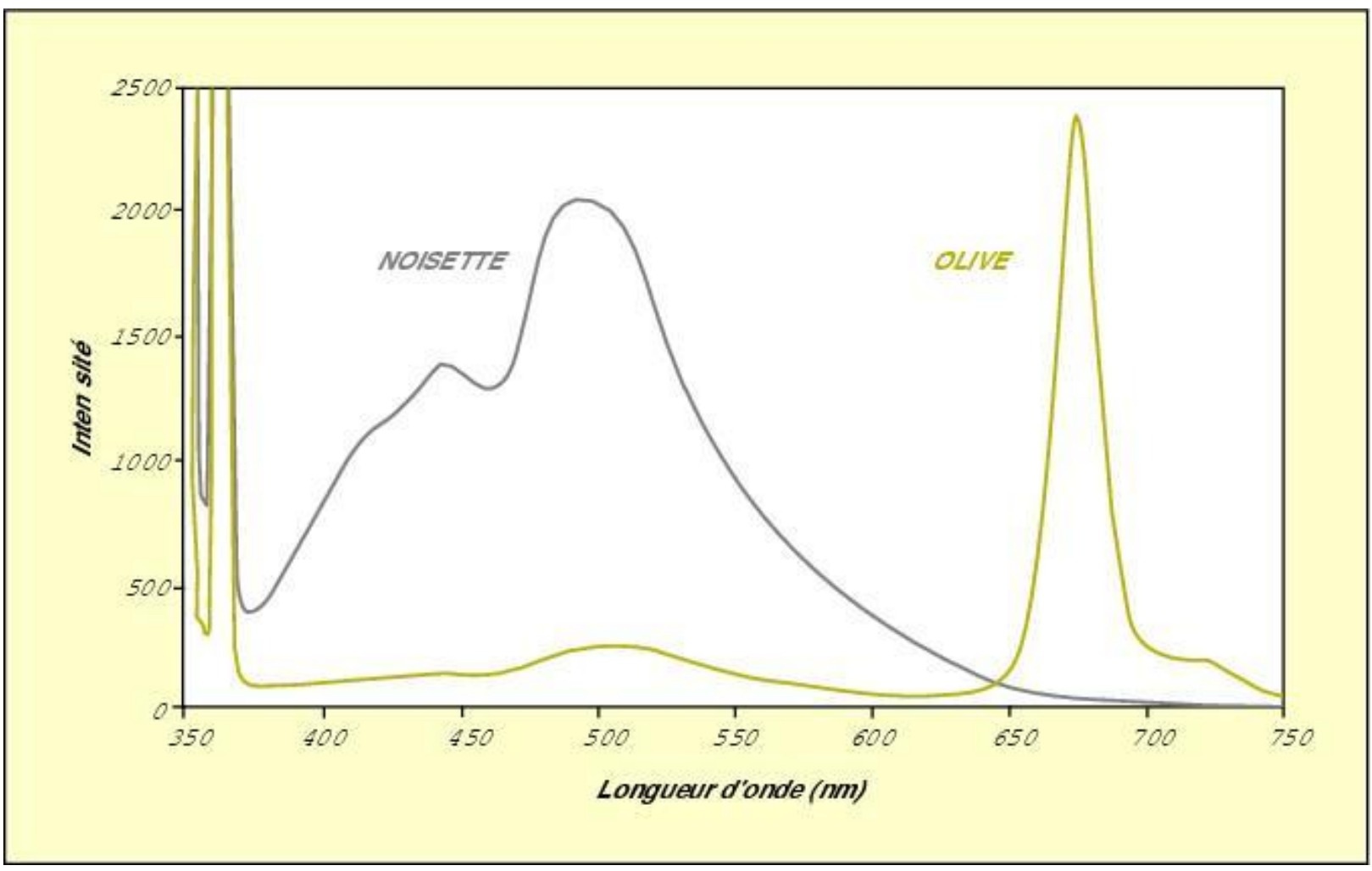

Figure 3. Spectres de fluorescence d'une huile de noisette et d'une huile d'olive. 


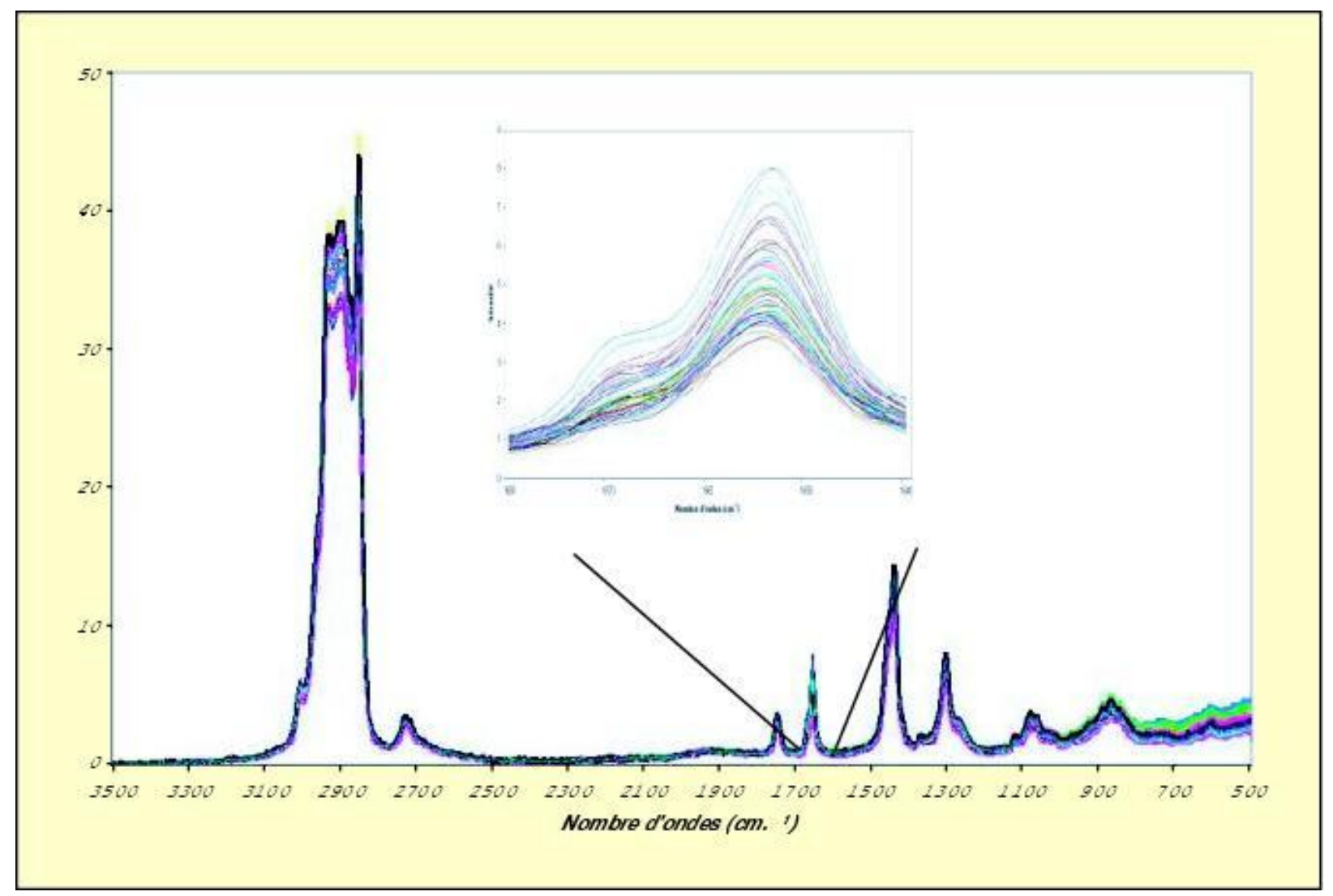

Figure 4. Spectres Raman de 80 échantillons de matière grasse laitière anhydre dont la zone comprise entre 1640 et $1680 \mathrm{~cm}^{-1}$ est présentée en fenêtre pour faire apparaître en détail les bandes caractéristiques des doubles liaisons cis $\left(1650 \mathrm{~cm}^{-1}\right)$ et trans $\left(1670 \mathrm{~cm}^{-1}\right)$ des acides gras insaturés. 